\title{
Changes of miR-155 expression in serum of uremic patients before and after treatment and risk factors analysis
}

\author{
HAO LI ${ }^{1}$, FANGXIN QIU $^{1}$, FANG TIAN ${ }^{1}$, XINZHENG SHI $^{1}$, AIQIN GAO $^{1}$, LEI SONG $^{2}$ and JUNLIANG LIU ${ }^{2}$ \\ ${ }^{1}$ Department of Nephrology and ${ }^{2}$ Intensive Care Unit, The People's Hospital of Chengyang, \\ Qingdao, Shandong 266109, P.R. China
}

Received October 16, 2019; Accepted May 20, 2020

DOI: $10.3892 /$ etm.2020.9067

\begin{abstract}
The aim of the present study was to investigate the changes of miR-155 expression in the serum of uremic patients before and after treatment and analyze the risk factors of efficacy. A total of 116 uremic patients admitted to the People's Hospital of Chengyang (Qingdao, China) were enrolled in the study as the uremia group, and were treated by hemodialysis combined with hemoperfusion, and 127 healthy subjects who underwent health examination during the same period were selected as the normal group. Reverse transcription quantitative PCR was used to detect the serum miR-155 levels of all the subjects in the two groups before treatment and those of uremia patients after treatment. The changes of clinically related indicators and inflammatory factors in uremic patients before and after treatment and their correlation with miR-155 were investigated. The risk factors affecting the efficacy of treatment were analyzed. Serum miR-155 levels in the uremia group were higher than those in the normal group $(\mathrm{P}<0.001)$; the miR-155 level in patients after treatment was significantly lower than that before treatment $(\mathrm{P}<0.001)$, and it was positively correlated with efficacy $(r=0.6873, \mathrm{P}<0.05)$. The serum miR-155 level in the invalid group was higher than that in the effective group, and the sensitivity and specificity of miR-155 for predicting the efficacy before treatment were 64.71 and $87.88 \%$, respectively. After treatment, the sensitivity and specificity of miR-155 for evaluating the efficacy were 76.47 and $88.89 \%$, respectively. Clinically related indicators and inflammatory factor levels in uremic patients decreased significantly after treatment, and the expression levels before and after treatment were significantly correlated with miR-155. Clinically related indicators, inflammatory factors and miR-155 were all risk factors affecting efficacy. The expression level of miR-155 in serum was significantly upregulated. Findings
\end{abstract}

Correspondence to: $\mathrm{Dr} \mathrm{Hao} \mathrm{Li}$, Department of Nephrology, The People's Hospital of Chengyang, 600 Changcheng Road, Qingdao, Shandong 266109, P.R. China

E-mail: hmt3mx@163.com

Key words: uremia, miR-155, hemodialysis combined with hemoperfusion, efficacy prediction, risk factors of this study suggest that monitoring miR-155 may reflect the efficacy and inflammatory state effectively.

\section{Introduction}

Chronic kidney disease is a global health problem. In recent years, the incidence of chronic kidney disease has been gradually increasing. In China, the overall prevalence rate of chronic kidney disease is estimated to be approximately $10.8 \%$ with a high mortality (1-3). Uremia belongs to the end-stage of chronic renal failure, which causes a series of clinical reactions and body system damages, seriously affecting patients' quality of life. If treatment is not timely, uremia caused by renal failure may aggravate the disease and eventually lead to death $(3,4)$. At present, findings have shown that hemodialysis combined with hemoperfusion has a good effect on the treatment of uremia $(5,6)$. Hemodialysis can remove blood urea nitrogen (BUN), creatinine ( $\mathrm{Cr}$ ) and bilirubin. In addition, it is mainly effective in removing internal wastes and adjusting acid-base balance through the principle of semipermeable membrane dialysis (7). Hemoperfusion can adsorb inflammatory mediators, such as interleukin (IL)-1, IL-6, IL-8, tumor necrosis factor- $\alpha$ and prothrombin-activating factor, to reduce inflammatory reaction (8-10). Hemodialysis combined with hemoperfusion can make up for each other and play different roles, thus achieving high efficiency in removing various toxins and maintaining water-electrolyte balance in the body $(5,7)$. Although blood purification technology and drug therapy have made progress, the mortality of chronic kidney disease remains very high (5).

MicroRNA (miRNA) is a small regulatory RNA with a length of 19-25 nucleotides that participates in posttranscriptional gene silencing in all eukaryotes (11). The miRNA role in a variety of kidney diseases, including nephrotic syndrome, renal fibrosis, lupus nephritis and acute pyelonephritis has been previously shown (12). In addition, previous findings have shown that miR-155 plays an important role in inflammation, immune response and formation of hematopoietic cells (13). It has been reported that miR-155 expression in liver tissues of patients with chronic liver diseases is upregulated, indicating that miR-155 may be influential in the progression of liver diseases, and it is a potential biomarker (14). Zhang et al (15) suggested that serum miR-155 may be regarded as a new target for early diagnosis and 
intervention of inflammatory state in uremic dialytic patients by detecting the changes of serum miR-155 level in those treated with alprostadil. However, clinical efficacy monitoring and the risk factors of miR-155 were not thoroughly studied.

By monitoring the changes of miR-155 expression in serum of uremic patients before and after treatment, the aim of the present study was mainly to explore the clinical value of miR-155 in hemodialysis combined with hemoperfusion treatment and the risk factors affecting their efficacy.

\section{Subjects and methods}

General information. A total of 116 uremic patients admitted to the People's Hospital of Chengyang (Qingdao, China) were selected as the uremia group, including 65 males and 51 females, with an average age of $51.32 \pm 8.97$ years. In addition, 127 healthy subjects who underwent health examination during the same period were selected as the normal group, including 69 males and 58 females, with an average age of $50.13 \pm 9.01$ years.

The study was approved by the Ethics Committee of the People's Hospital of Chengyang (no. CYPH2017011A), and all the patients and their families were informed prior to the study and signed a complete informed consent form.

Inclusion criteria were as follows: Uremic patients who met the disease diagnosis and were in relatively stable condition; patients who showed good compliance and had complete clinical data; patients who received routine examinations, such as urine routine test, blood routine test, electrocardiogram; patients with normal liver and kidney function after admission; patients who were accompanied by their family when admitted to the hospital. All subjects that participated in the study signed an informed consent form.

Exclusion criteria were as follows: Patients with liver dysfunction, severe organ lesions, history of craniocerebral trauma, autoimmune system defects, or mental hereditary diseases; patients who did not actively cooperate or had habits harmful to the health such as long-term smoking and alcoholism.

Treatment plan. Patients with uremia were treated routinely after admission; in order to avoid bad eating habits, their blood pressure and blood sugar were controlled, and they received other symptomatic and supportive treatment. They were treated via hemodialysis combined with hemoperfusion. Hemodialysis was conducted using a German Fressnius 4008S type hemodialysis machine, and anticoagulation was conducted by carbonate dialysate and low molecular heparin sodium, with a surface area of $1.3 \mathrm{~m}^{2}$, ultrafiltration coefficient of $40 \mathrm{ml} / \mathrm{h} \mathrm{mmHg}$, blood flow of 200-250 ml/min, and dialysate flow of $500 \mathrm{ml} / \mathrm{min}, 4 \mathrm{~h}$ each time, 3 times a week. Boxin MG 150 disposable hemoperfusion device was used, and the perfusion device was connected in series to the front end of the dialyzer. Two hours after treatment, the perfusion device was taken down after its adsorption capacity was saturated, hemodialysis was performed for $2 \mathrm{~h}$ separately, and joint treatment was performed once a week. Relevant indicators were compared 12 weeks after treatment. In addition, adequate sleep, limited water, low salt and low fat diet were ensured during the treatment of patients.
Detection of serum miR-155. In the morning, $4 \mathrm{ml}$ of fasting elbow venous blood was collected from the subjects in the two groups after admission as well as uremia patients after treatment, respectively. The collected blood was placed in a centrifuge tube to collect upper serum, centrifugation conditions were as follows: $1006.2 \mathrm{x} \mathrm{g}, 10 \mathrm{~min}, 10 \mathrm{~cm}$ (radius), $4^{\circ} \mathrm{C}$ (temperature), and then it was placed in a refrigerator at $-80^{\circ} \mathrm{C}$ for testing. Total RNA in serum was extracted by TRIzol ${ }^{\circledR}$ kit (Invitrogen) according to the manufacturer's instructions, and the template RNA was digested and treated with DNaseI to eliminate DNA contamination. An ultraviolet spectrophotometer (Beijing UP General Technology Co., Ltd.) was used to measure the purity and concentration; the RNA sample was then reverse transcribed into cDNA, and the procedure was strictly carried out in accordance with the instructions of cDNA reverse transcription kit (Takara). Reverse transcription-quantitative PCR (RT-qPCR) was used for detection, and SYBR PrimeScript miRNA RT-qPCR kit (Takara) was used for RT-qPCR in ABI Prism 7500 (Applied Biosystems). PCR reaction conditions were as follows: Enzyme activation at $95^{\circ} \mathrm{C}$ for $10 \mathrm{~min}$, denaturation at $95^{\circ} \mathrm{C}$ for $15 \mathrm{sec}$, annealing/extension at $60^{\circ} \mathrm{C}$ for $1 \mathrm{~min}$, a total of 40 cycles. Primers for this experiment were designed by Primer Premier 5.0 (Premier), and generated by Tianjin Saier Biotechnology Co., Ltd. U6 was used as internal reference, and the specific primer sequences are shown in Table I. The above system configuration was strictly in accordance with the instructions, and the content of miR-155 was calculated by $2^{-\Delta \Delta \mathrm{Cq}}(16)$.

Efficacy evaluation $(17,18)$. Scoring for efficacy evaluation was as follows: Marked effect: After treatment, clinical symptoms and signs of patients significantly improved; improvement: The clinical symptoms and signs of patients improved after relevant treatment, but these were not significant; invalid: Patients' clinical symptoms and signs did not change or deteriorate significantly after relevant treatment.

Outcome measures. Clinically related indicators of uremic patients before and after treatment were observed, including BUN, Cr, $\beta 2$-microglobulin ( $\beta 2-\mathrm{MG})$, parathyroid hormone (PTH), C-reactive protein (CRP) and IL-6. The changes of serum miR-155 level and evaluation of the efficacy on uremic patients were observed to analyze the risk factors affecting their efficacy.

Statistical analysis. SPSS 20.0 (IBM Corp., Armonk) was used for statistical analysis, and GraphPad Prism 7 (San Diego Graphpad Software Co., Ltd.) was used to draw images of the collected data. The counting data were expressed as [n (\%)], and the Chi-square test was used for inter-group comparison. The measurement data were expressed as mean \pm standard deviation (SD), independent-samples t-test and paired sample t-test were used for comparison between the two groups before and after therapy, and receiver operating characteristic curve (ROC) was used to predict and evaluate the efficacy of uremia patients. Pearson's correlation coefficient was used for bivariate normal distribution data, Spearman's correlation coefficient was used to analyze the correlation between grade and variable, and the multivariate logistic regression was used to analyze the risk factors affecting their treatment, with statistical significance $(\mathrm{P}<0.05)$. 
Table I. Primer sequences.

\begin{tabular}{lll}
\hline Genes & \multicolumn{1}{c}{ Upstream } & \multicolumn{1}{c}{ Downstream } \\
\hline miR-155 & 5'-GTGCAGGGTCCGAGGT-3' & 5'-CGCTTAATGCTAATCGTGATAGG-3' \\
U6 & 5'-CTCGCTTCGGCAGCACA-3' & 5'-AACGCTTCACGAATTTGCGT-3' \\
\hline
\end{tabular}

\section{Results}

Comparison of clinical general data. Statistical comparison of clinical general data between the two groups is shown in Table II. There was no significant difference with regard to sex, age, average age and body mass index (BMI) between the uremia and normal groups $(\mathrm{P}>0.05)$. Hemoglobin and blood albumin in uremia group were lower than those in normal group $(\mathrm{P}<0.05)$, whereas total cholesterol, triglyceride, low-density lipoprotein and mean arterial pressure in uremia group were higher than those in normal group $(\mathrm{P}<0.05)$.

Evaluation of efficacy of changes in serum miR-155 level on uremic patients. According to the detection of serum miR-155 levels in the two groups, the serum miR-155 level in uremic patients was significantly higher than that in the normal group (Fig. 1A, P<0.001), and that in uremic patients after treatment was significantly lower than before treatment (Fig. 1B, P<0.001); there were 43 markedly effective cases, 56 improved cases and 17 invalid cases after treatment. As is evident in Fig. 1C, the miR-155 level was significantly positively correlated with the efficacy $(\mathrm{r}=0.6873, \mathrm{P}<0.05)$; the worse the efficacy was, the higher the miR-155 level was. In addition, according to the efficacy, patients were divided into effective group $(n=99)$ and invalid group $(n=17)$; the serum miR-155 level of patients in the invalid group was significantly higher than that in the effective group $(\mathrm{P}<0.001$; Fig. 1D). ROC curve was drawn according to miR-155 level before and after treatment; area under the curve (AUC) of miR-155 level used for predicting efficacy of uremic patients before treatment was 0.788 , sensitivity was $64.71 \%$ and specificity was $87.88 \%$ (Fig. 1E); AUC of miR-155 level for evaluating efficacy of uremic patients after treatment was 0.895 , sensitivity was $76.47 \%$ and specificity was $88.89 \%$ (Fig. 1F).

Changes of clinically related indicators. The levels of serum clinically related indicators and inflammatory factors in uremic patients before and after treatment were detected; 32-MG, Cr, BUN, PTH, CRP, and IL-6 levels after treatment were significantly lower than those before treatment, with statistically significant difference ( $\mathrm{P}<0.001$; Fig. 2$)$.

Correlation between serum miR-155 level and clinical related indicators

Correlation between serum miR-155 level and clinical related indicators before treatment. Pearson's correlation coefficient analysis showed that prior to treatment, serum miR-155 level was significantly positively correlated with clinically related indicators such as $\beta 2-\mathrm{MG}, \mathrm{Cr}, \mathrm{BUN}$ and $\mathrm{PTH}(\mathrm{P}<0.001$; Fig. 3A-D), as well as with levels of CRP and IL-6 inflammatory factors $(\mathrm{P}<0.001$; Fig. 3E and $\mathrm{F})$.
Correlation between serum miR-155 level and clinically related indicators after treatment. Pearson's correlation coefficient analysis revealed that after treatment, serum miR-155 level was significantly positively correlated with clinically related indicators such as $\beta 2-\mathrm{MG}, \mathrm{Cr}, \mathrm{BUN}$ and $\mathrm{PTH}$ $(\mathrm{P}<0.001$; Fig. 4A-D), as well as with levels of CRP and IL-6 inflammatory factors $(\mathrm{P}<0.001$; Fig. 4E and $\mathrm{F})$.

Analysis on influencing factors of efficacy. According to the efficacy, uremic patients were divided into the effective $(n=99)$ and invalid $(n=17)$ groups and were assessed via univariate analysis. There were differences in $\beta 2-\mathrm{MG}, \mathrm{Cr}$, BUN, PTH, CRP, IL-6, and miR-155 levels between the two groups, as shown in Table III $(\mathrm{P}<0.05)$. Results of Logistic regression analysis on risk factors indicated that $\beta 2-\mathrm{MG}$ (HR: 7.705, 95\% CI: 1.380-43.030), Cr (HR: 6.979, 95\% CI: 0.921-52.906), BUN (HR: 6.869, 95\% CI: 1.042-45.281), PTH (HR: $6.859,95 \%$ CI: 1.046-44.995), CRP (HR: 7.901, 95\% CI: 1.202-51.952), IL-6 (HR: 7.319, 95\% CI: 1.036-51.687), and miR-155 (HR: 9.562, 95\% CI: 1.520-60.163) were all risk factors affecting their efficacy. More details are provided in Table IV.

\section{Discussion}

Uremia mainly results from the decline of glomerular filtration function which causes acidolysis disorder, water electrolyte disorder and other phenomena. It leads to the toxic metabolites retention phenomenon, thus affecting blood, digestive tract and nerve, resulting in severe systemic symptoms $(19,20)$. Recently, with the popularization and development of blood purification technology, good news has been afforded to patients with end-stage renal disease, and their quality of life has been significantly improved $(5,21)$. However, various factors in the process of hemodialysis still seriously affect the efficacy of maintenance hemodialysis patients, and the experiment of this study further clarified the diagnosis and treatment experience of uremia by analyzing the changes of miR-155 in the treatment process.

Findings have shown that triglyceride, total cholesterol, blood glucose, and low-density lipoprotein cholesterol increased significantly in patients with chronic kidney disease (22). Previous results have shown that hemoglobin and albumin in uremic patients were lower than those in healthy individuals (15). Those results were similar to the results of this study, showing that the occurrence of the disease may lead to the disorder of lipid and blood metabolism to some extent. Additionally, miR-155 upregulated significantly in clear cell renal cell carcinoma tissues compared with corresponding normal tissues (23). The miR-155 expression in peripheral blood mononuclear cells of patients with rheumatoid arthritis 
Table II. Comparison of general clinical data between the two groups [n (\%), mean \pm SD].

\begin{tabular}{|c|c|c|c|c|}
\hline Clinical data & Uremia group $(n=116)$ & Normal group $(n=127)$ & $\mathrm{F} / \chi^{2}$ & P-value \\
\hline Gender & & & 0.071 & 0.790 \\
\hline Male & $65(56.03)$ & $69(54.33)$ & & \\
\hline Female & $51(43.97)$ & $58(45.67)$ & & \\
\hline Age, years & & & 0.078 & 0.780 \\
\hline$<50$ & $50(43.10)$ & $57(44.88)$ & & \\
\hline$\geq 50$ & $66(56.90)$ & $70(55.12)$ & & \\
\hline Average age (years) & $51.32 \pm 8.97$ & $50.13 \pm 9.01$ & 1.031 & 0.963 \\
\hline $\operatorname{BMI}\left(\mathrm{kg} / \mathrm{m}^{2}\right)$ & $24.01 \pm 3.43$ & $23.89 \pm 3.13$ & 0.285 & 0.315 \\
\hline Hemoglobin (g/l) & $88.72 \pm 15.49$ & $144.38 \pm 12.89$ & 30.540 & $<0.05$ \\
\hline Blood albumin (g/l) & $36.14 \pm 5.29$ & $45.21 \pm 4.45$ & 14.500 & $<0.05$ \\
\hline Total cholesterol (mmol/l) & $5.08 \pm 1.09$ & $4.15 \pm 1.32$ & 5.956 & $<0.05$ \\
\hline Triglycerides (mmol/l) & $1.82 \pm 0.68$ & $1.02 \pm 0.51$ & 10.430 & $<0.05$ \\
\hline Low-density lipoprotein (mmol/l) & $3.05 \pm 0.82$ & $2.31 \pm 0.87$ & 6.807 & $<0.05$ \\
\hline Mean arterial pressure $(\mathrm{mmHg})$ & $112.37 \pm 10.01$ & $92.35 \pm 8.07$ & 17.230 & $<0.05$ \\
\hline
\end{tabular}

BMI, body mass index.
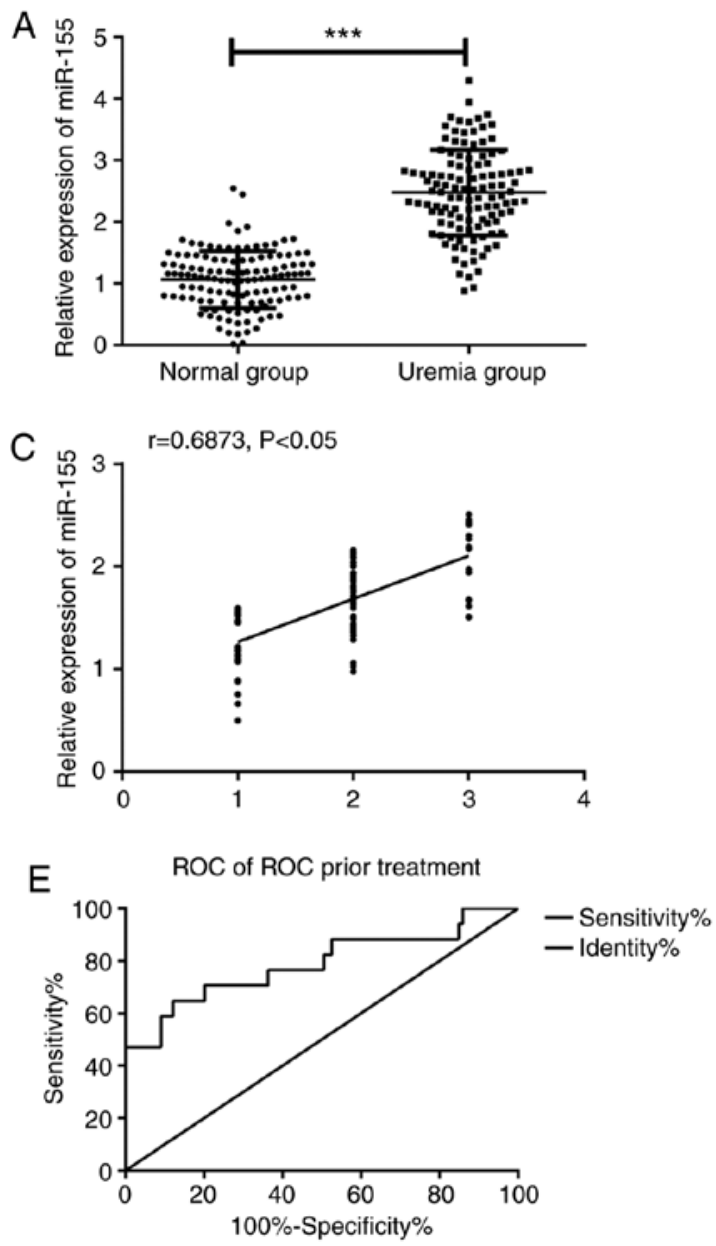

B
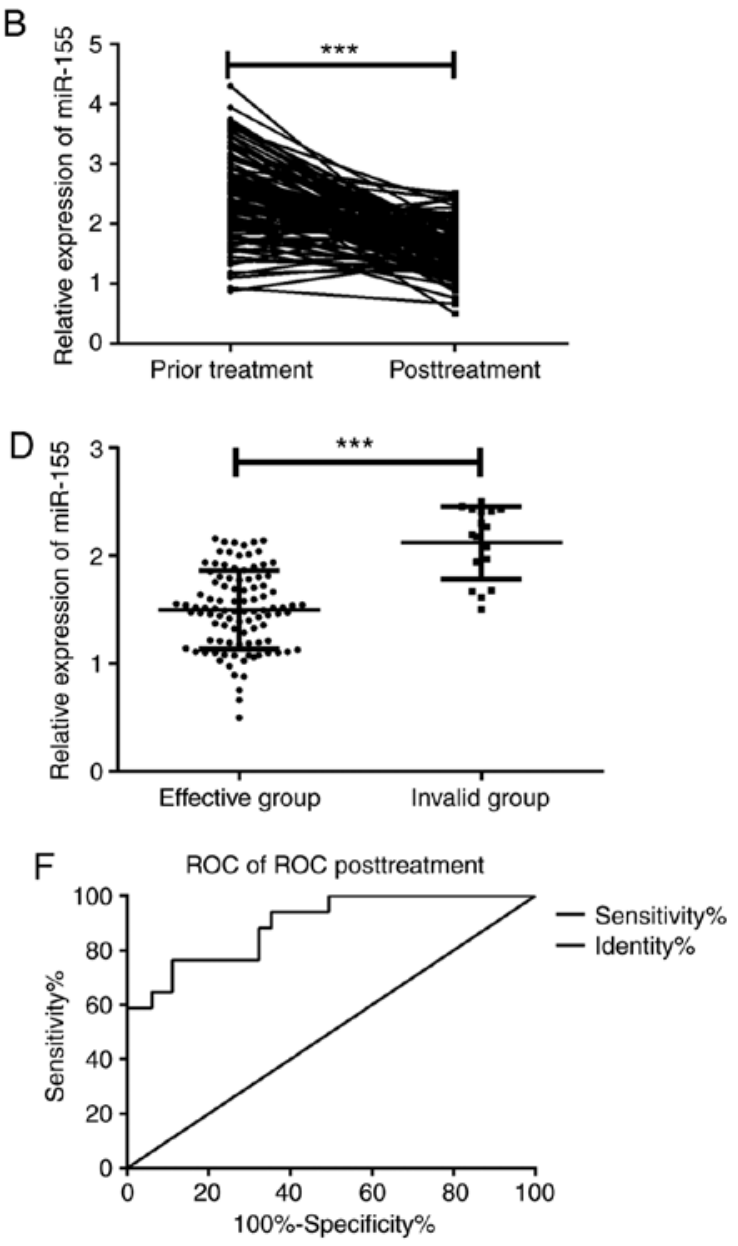

Figure 1. Evaluation of efficacy of changes in serum miR-155 level on uremic patients. (A) The serum miR-155 level in uremic patients was significantly higher than that in normal group. (B) The miR-155 level in uremic patients after treatment was significantly lower than that before treatment. (C) Spearman correlation coefficient was used to analyze the correlation between the miR-155 level and efficacy, and it showed that the miR-155 level was significantly positively correlated with efficacy $(\mathrm{r}=0.6873, \mathrm{P}<0.05)$. (D) The serum miR-155 level in the invalid group was significantly higher than that in the effective group. (E) AUC of miR-155 level for predicting efficacy of uremic patients prior to treatment was 0.788 , sensitivity was $64.71 \%$ and specificity was $87.88 \%$. (F) AUC of miR-155 level for evaluating efficacy of uremic patients after treatment was 0.895 , sensitivity was $76.47 \%$ and specificity was $88.89 \%$. Abscissa 1 in Fig. 1C indicated marked effect, 2 indicated improvement, and 3 indicated invalid. ${ }^{* * *} \mathrm{P}<0.001$. 
A

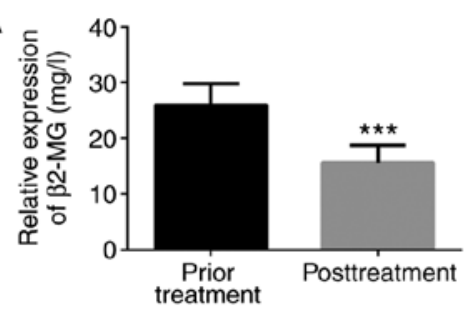

D

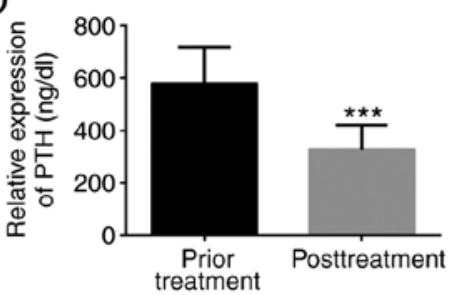

B

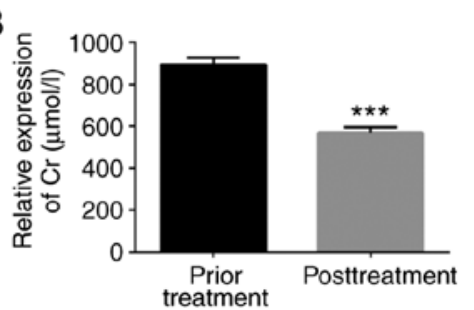

E

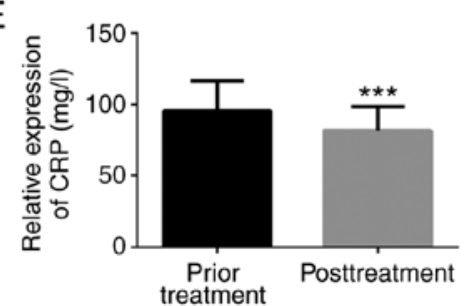

C

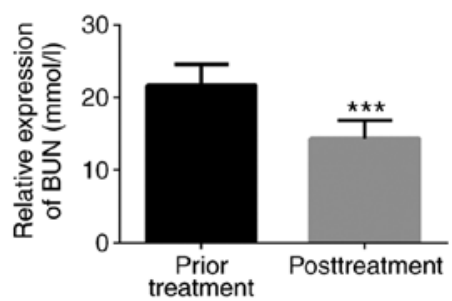

$\mathrm{F}$

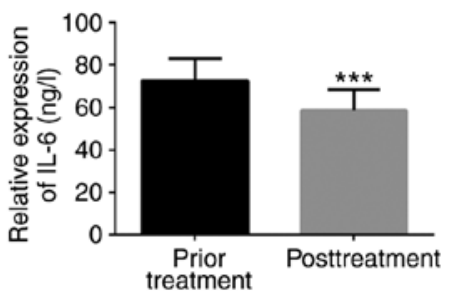

Figure 2. Changes of clinically related indicators. (A) The $\beta 2-\mathrm{MG}$ level after treatment was lower than that before treatment. (B) The Cr level after treatment was lower than that before treatment. (C) The BUN level after treatment was lower than that before treatment. (D) The PTH level after treatment was lower than that before treatment. (E) The CRP level after treatment was lower than that before treatment. (F) The level of IL-6 after treatment was lower than that before treatment. ${ }^{* * * *} \mathrm{P}<0.001$, compared with before treatment.
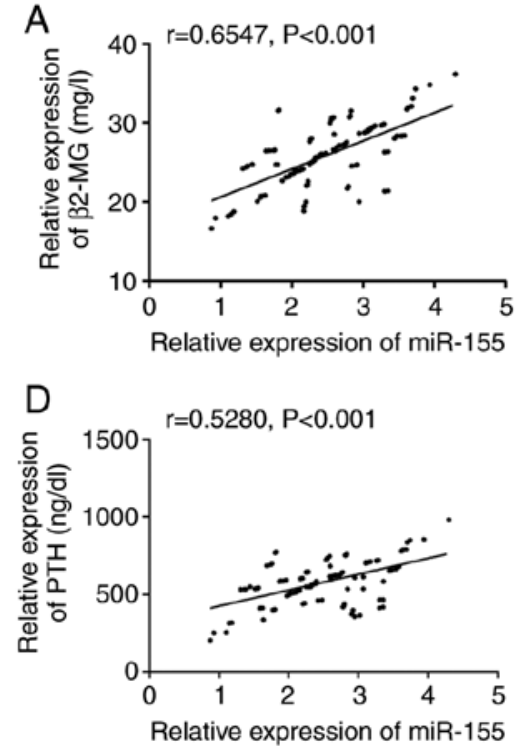

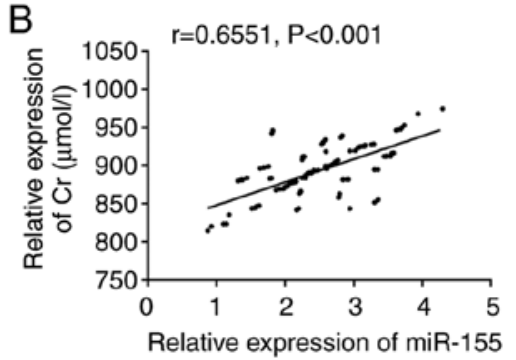

$\mathrm{E}$

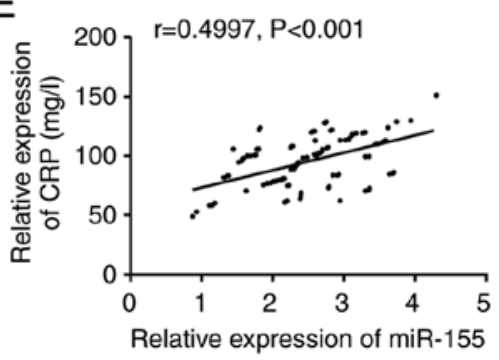

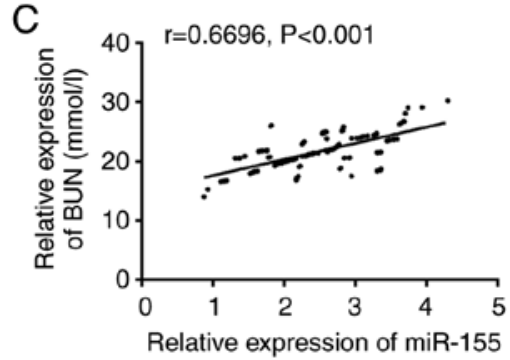

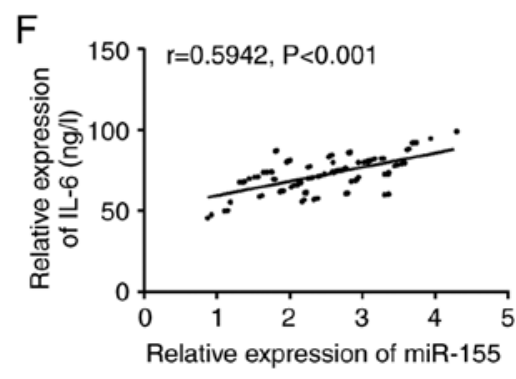

Figure 3. Correlation between serum miR-155 level and clinically related indicators before treatment. (A) miR-155 was positively correlated with $\beta 2-\mathrm{MG}$ before treatment $(\mathrm{r}=0.6547, \mathrm{P}<0.001)$. (B) Before treatment, miR-155 was positively correlated with $\mathrm{Cr}(\mathrm{r}=0.6551, \mathrm{P}<0.001)$. $(\mathrm{C}) \mathrm{Before}$ treatment, miR-155 was positively correlated with BUN ( $\mathrm{r}=0.6696, \mathrm{P}<0.001)$. (D) Before treatment, miR-155 was positively correlated with $\mathrm{PTH}$ ( $\mathrm{r}=0.5280$, $\mathrm{P}<0.001)$. $(\mathrm{E}) \mathrm{Before}$ treatment, miR-155 was positively correlated with CRP $(\mathrm{r}=0.4997, \mathrm{P}<0.001)$. (F) Before treatment, miR-155 was positively correlated with IL-6 ( $\mathrm{r}=0.5942$, $\mathrm{P}<0.001)$.

also increased significantly, and it indicated that miR-155 had a correlation with the activity of the disease (24). The current study examined the serum miR-155 level of uremic patients and normal subjects, and the results showed that the level in the former was significantly higher than that in the latter, which was similar to the results of Zhang et al (15). We further observed the changes of miR-155 level before and after treatment and found that the level of uremic patients after treatment was significantly lower than that before treatment, suggesting that hemodialysis combined with hemoperfusion treatment could significantly lower miR-155 level. Nevertheless, there was still doubt as to whether its expression was linked to the efficacy. In addition, we found that the miR-155 level after treatment was significantly positively correlated with the efficacy through Spearman's correlation analysis, and that of patients in the invalid group was significantly higher than that in the effective group; the worse the efficacy was, the higher the miR-155 level was. The results suggested that miR-155 may be used as a therapeutic indicator for uremic patients. Monitoring the level of miR-155 can reflect the state of an illness in time, which has a certain clinical value in replacing treatment plans without delay. Ulivi et al (25) suggested that serum miR-20b, miR-29b, and miR-155 levels could be used as efficacy predictors of bevacizumab in patients with metastatic colorectal cancer, and were correlated with disease progression-free survival and overall survival rate. In this study, ROC curves were drawn 
A
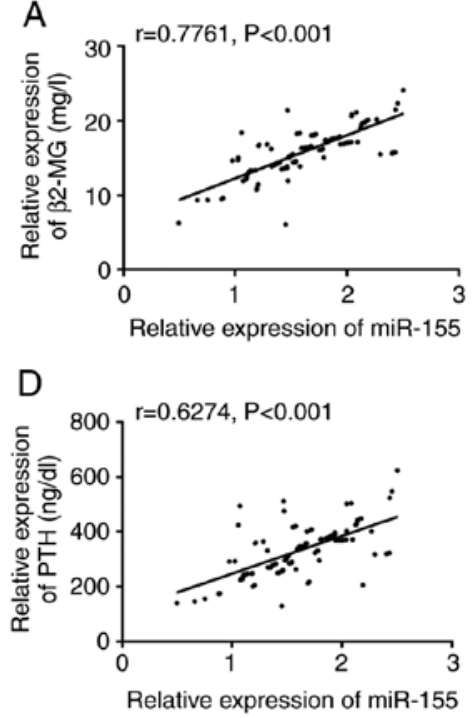

B

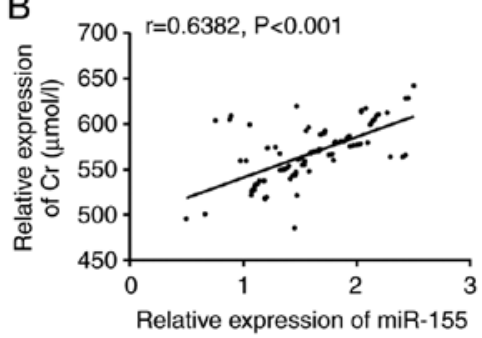

E

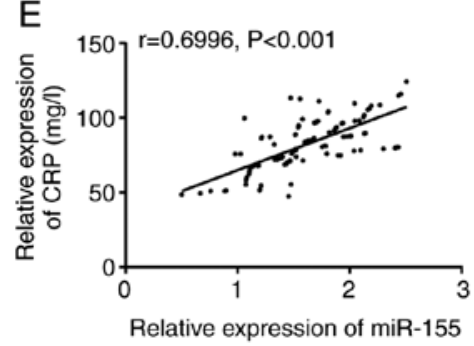

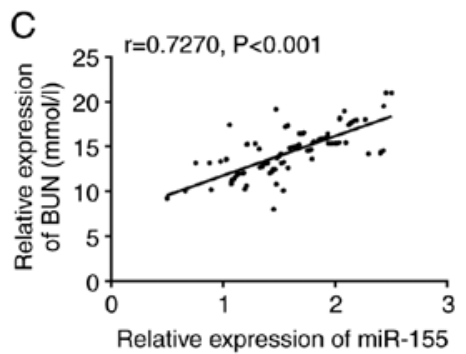

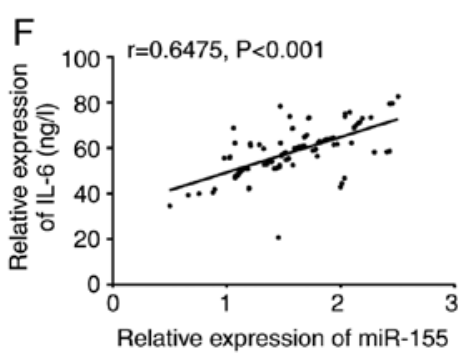

Figure 4. Correlation between serum miR-155 level and clinically related indicators after treatment. (A) After treatment, miR-155 level was positively correlated with $\beta 2-\mathrm{MG}(\mathrm{r}=0.7761, \mathrm{P}<0.001)$. (B) After treatment, miR-155 level was positively correlated with $\mathrm{Cr}(\mathrm{r}=0.6382, \mathrm{P}<0.001)$. $(\mathrm{C}) \mathrm{After}$ treatment, miR-155 level was positively correlated with BUN ( $\mathrm{r}=0.7270, \mathrm{P}<0.001)$. (D) After treatment, miR-155 level was positively correlated with $\mathrm{PTH}(\mathrm{r}=0.6274$, $\mathrm{P}<0.001)$. (E) After treatment, miR-155 level was positively correlated with $\mathrm{CRP}(\mathrm{r}=0.6996, \mathrm{P}<0.001)$. (F) After treatment, miR-155 level was positively correlated with IL-6 ( $\mathrm{r}=0.6475, \mathrm{P}<0.001)$.

according to miR-155 levels before and after treatment, and it was found that those before and after treatment could be used to predict and evaluate the efficacy of uremic patients, and were expected to become biomarkers. Nevertheless, more studies are needed to prove the relationship between miR-155 level and long-term survival of uremic patients. Compared with previous studies, Liang et al (26) observed that the levels of miR-34a, miR-205, and miR-155 in urine of patients with IgA nephropathy were significantly lower than those of normal subjects. Then, they were divided into complete sustained-release group and incomplete sustained-release group according to efficacy. Their findings indicated there was no remarkable difference in the miR-155 levels in urine of the two groups $(\mathrm{P}>0.05)$. Furthermore, it has been reported that a high miR-155 expression was an independent predictor of lower complete-sustained release rate and shorter overall survival in patients with acute myeloid leukemia (27). Combined with this study, it was suggested that the miR-155 expression was different in varying diseases and samples, its abnormal expression could be used for the clinical monitoring of various diseases, and its detection and prediction value in chronic kidney disease still required further research.

$\beta 2-\mathrm{MG}$ and PTH belong to macromolecular toxins; when the human body is in an inflammatory state, it accelerates the production and release of $\beta 2-\mathrm{MG}$, mainly through renal degradation and re-absorption into the body $(28,29)$. Therefore, the concentration of $\beta 2-\mathrm{MG}$ can be used to reflect the filtration function of the kidney. Accumulation of $\beta 2-\mathrm{MG}$ and metabolic disorder of uremic patients may produce a large amount of $\mathrm{PTH}$, which is involved in the occurrence and development of renal diseases $(29,30)$. $\mathrm{Cr}$ and BUN belong to small molecular toxins and are one of the important factors causing uremic symptoms and complications $(7,31,32)$. Inflammation is a vital factor that causes the occurrence or aggravation of various diseases, and is also crucial in the pathogenesis of complications in uremic patients (33). IL- 6 and CRP are important members of inflammatory cytokines, have strong biological effects and can regulate cellular immune response, and upregulate when the body is in a pathological state $(34,35)$. In this study, the levels of serum clinically related indicators and inflammatory factors in uremic patients before and after treatment were detected, and it was found that the $\beta 2-\mathrm{MG}, \mathrm{Cr}, \mathrm{BUN}, \mathrm{PTH}, \mathrm{CRP}$, and IL-6 levels after treatment were significantly lower than those prior to treatment. This showed that hemodialysis combined with hemoperfusion could effectively remove toxins and improve the inflammatory state of uremic patients, which was consistent with the results of previous studies $(6,31,36)$; hemodialysis combined with hemoperfusion had a high value in treating uremia. The Pearson correlation coefficient analysis revealed that before treatment, serum miR-155 level was significantly positively correlated with clinically related indicators of $\beta 2-\mathrm{MG}, \mathrm{Cr}, \mathrm{BUN}$, and $\mathrm{PTH}(\mathrm{P}<0.001)$, and it was significantly positively correlated with levels of CRP and IL-6 inflammatory factors $(\mathrm{P}<0.001)$. The results further verified that miR-155 were correlated with disease-related indicators of uremic patients, could effectively reflect their disease state, and provided a new target for intervention or treatment thereof. At the same time, combined with previous studies, miR-155 further confirmed that miR-155 was able to participate in the pathophysiological process of inflammatory state of uremic patients by regulating the expression levels of IL-6 and CRP (15). It was reported that proteinuria $\geq 1.0 \mathrm{~g} / 24 \mathrm{~h}$, high systolic pressure, and diastolic pressure were the risk factors affecting the improvement of blood pressure control in patients with chronic kidney disease by logistic regression analysis (37). Wang et al (38) pointed out that a high level of miR-155 was an independent risk factor for poor prognosis and lymphocyte infiltration in non-small cell lung cancer. To the best of our knowledge, there is currently no study on the 
Table III. Univariate analysis $[\mathrm{n}(\%)$, mean $\pm \mathrm{SD}]$.

\begin{tabular}{|c|c|c|c|c|}
\hline Variables & Effective group $(\mathrm{n}=99)$ & Invalid group $(n=17)$ & $t / \chi^{2}$ & P-value \\
\hline Gender & & & 0.063 & 0.802 \\
\hline Male & $55(55.56)$ & $10(58.82)$ & & \\
\hline Female & $44(44.44)$ & $7(41.18)$ & & \\
\hline Age, years & & & 0.910 & 0.340 \\
\hline$<50$ & $45(45.45)$ & $5(29.41)$ & & \\
\hline$\geq 50$ & $54(54.55)$ & $12(70.59)$ & & \\
\hline BMI $\left(\mathrm{kg} / \mathrm{m}^{2}\right)$ & $23.93 \pm 3.09$ & $24.09 \pm 3.12$ & 0.401 & 0.689 \\
\hline$\beta 2-\mathrm{MG}(\mathrm{mg} / \mathrm{l})$ & $15.02 \pm 2.74$ & $19.30 \pm 2.70$ & 12.260 & $<0.05$ \\
\hline $\mathrm{Cr}(\mu \mathrm{mol} / \mathrm{l})$ & $563.59 \pm 28.90$ & $594.15 \pm 24.37$ & 11.860 & $<0.05$ \\
\hline $\mathrm{BUN}(\mathrm{mmol} / \mathrm{l})$ & $13.82 \pm 2.23$ & $17.48 \pm 1.90$ & 13.810 & $<0.05$ \\
\hline PTH (ng/dl) & $308.64 \pm 76.88$ & $444.64 \pm 86.85$ & 12.876 & $<0.05$ \\
\hline CRP (mg/l) & $79.26 \pm 15.84$ & $95.84 \pm 15.82$ & 8.155 & $<0.05$ \\
\hline IL-6 (ng/l) & $56.42 \pm 8.89$ & $70.66 \pm 7.53$ & 13.510 & $<0.05$ \\
\hline miR-155 level & $1.55 \pm 0.42$ & $1.80 \pm 0.42$ & 4.635 & $<0.05$ \\
\hline
\end{tabular}

BMI, body mass index; $\beta 2-\mathrm{MG}, \beta 2$-microglobulin; Cr, creatinine; BUN, blood urea nitrogen; PTH, parathyroid hormone; CRP, C-reactive protein; IL, interleukin.

Table IV. Multivariate analysis.

\begin{tabular}{|c|c|c|c|c|c|}
\hline Variables & $\beta$ & SD & $\chi^{2}$ & P-value & HR $(95 \% \mathrm{CI})$ \\
\hline$\beta 2-\mathrm{MG}(\mathrm{mg} / \mathrm{l})$ & 2.042 & 0.878 & 5.414 & 0.020 & $7.705(1.380-43.030)$ \\
\hline $\mathrm{Cr}(\mu \mathrm{mol} / \mathrm{l})$ & 1.943 & 1.034 & 3.534 & 0.048 & $6.979(0.921-52.906)$ \\
\hline BUN (mmol/l) & 1.927 & 0.962 & 4.011 & 0.045 & $6.869(1.042-45.281)$ \\
\hline PTH (ng/dl) & 1.926 & 0.960 & 4.026 & 0.045 & $6.859(1.046-44.995)$ \\
\hline CRP (mg/l) & 2.067 & 0.961 & 4.627 & 0.031 & $7.901(1.202-51.952)$ \\
\hline IL-6 (ng/l) & 1.990 & 0.997 & 3.983 & 0.046 & $7.319(1.036-51.687)$ \\
\hline miR-155 level & 2.258 & 0.938 & 5.789 & 0.016 & $9.562(1.520-60.163)$ \\
\hline
\end{tabular}

$\beta 2-\mathrm{MG}, \beta 2$-microglobulin; Cr, creatinine; BUN, blood urea nitrogen; PTH, parathyroid hormone; CRP, C-reactive protein; IL, interleukin; $\mathrm{HR}$, hazard ratio; CI, confidence interval.

risk factors for efficacy of uremic patients. The present study employed logistic regression analysis on risk factors, and the results showed that high levels of $\beta 2-\mathrm{MG}, \mathrm{Cr}, \mathrm{BUN}, \mathrm{PTH}$, CRP, IL-6, and miR-155 were all risk factors affecting their efficacy. Close attention should be given to the changes of the above indicators during uremia treatment to provide some basis for changing the treatment plans.

In this study, the changes of miR-155 expression in serum of uremic patients before and after treatment were detected, and the risk factors affecting their efficacy were analyzed. However, this study has certain limitations, as it only evaluates the efficacy based on the basic efficacy time and does not observe the long-term effects. Moreover, due to the lack of in vitro experiments, the mechanism of miR-155 on uremia is still at the stage of speculation. In future research, rigorous experimental analysis should be carried out as early as possible to obtain the optimal experimental results against the above limitations.
To sum up, the expression level of miR-155 in serum of uremic patients was upregulated significantly and monitoring miR-155 can effectively reflect its efficacy and inflammatory state.

\section{Acknowledgements}

Not applicable.

\section{Funding}

No funding was received.

\section{Availability of data and materials}

The datasets used and/or analyzed during the present study are available from the corresponding author on reasonable request. 


\section{Authors' contributions}

HL conceived the study and wrote the manuscript. FQ and FT analyzed and interpreted the patient general data. XS and AG performed PCR. LS and JL were responsible for the analysis of the observation indicators. All authors read and approved the final version of the manuscript.

\section{Ethics approval and consent to participate}

The study was approved by the Ethics Committee of The People's Hospital of Chengyang (Qingdao, China). The patients who participated in this research had complete clinical data. All patients and their families were informed prior to the study and signed a complete informed consent form.

\section{Patient consent for publication}

Not applicable.

\section{Competing interests}

The authors declare that they have no competing interests.

\section{References}

1. Scherer A, Günther OP, Balshaw RF, Hollander Z Wilson-McManus J, Ng R, McMaster WR, McManus BM and Keown PA: Alteration of human blood cell transcriptome in uremia. BMC Med Genomics 6: 23, 2013.

2. Chapagain A, Caton PW, Kieswich J, Andrikopoulos P Nayuni N, Long JH, Harwood SM, Webster SP, Raftery MJ, Thiemermann $\mathrm{C}$, et al: Elevated hepatic $11 \beta$-hydroxysteroid dehydrogenase type 1 induces insulin resistance in uremia. Proc Natl Acad Sci USA 111: 3817-3822, 2014.

3. Shang W, Shen Y, Gao S, Feng G, Feng Y, Wang Z and Zhang X: Comparison of HLA-A, -B and -DRB1 loci polymorphism between kidney transplants of uremia patients and healthy individuals in Central China. PLoS One 11: e0165426, 2016.

4. Vitetta L and Gobe G: Uremia and chronic kidney disease: The role of the gut microflora and therapies with pro- and prebiotics. Mol Nutr Food Res 57: 824-832, 2013.

5. Lu W and Jiang GR; HD/HP versus HD trial Group: Randomised, open-label, multicentre trial comparing haemodialysis plus haemoperfusion versus haemodialysis alone in adult patients with end-stage renal disease (HD/HP vs. HD): Study protocol. BMJ Open 8: e022169, 2018.

6. Li J, Li D, Xu Y, Wang A, Xu C and Yu C: The optimal timing of hemoperfusion component in combined hemodialysis-hemoperfusion treatment for uremic toxins removal. Ren Fail 37: 103-107, 2015.

7. Li Z, Wang G, Zhen G, Zhang Y, Liu J and Liu S: Effects of hemodialysis combined with hemoperfusion on severe acute pancreatitis. Turk J Gastroenterol 29: 198-202, 2018.

8. Wang YT, Fu JJ, Li XL, Li YR, Li CF and Zhou CY: Effects of hemodialysis and hemoperfusion on inflammatory factors and nuclear transcription factors in peripheral blood cell of multiple organ dysfunction syndrome. Eur Rev Med Pharmacol Sci 20: $745-750,2016$.

9. Xu X, Yu Z, Liang Y, Gao H, Liu Y and Yu Y: Mumps caused by paraquat-induced poisoning: A case report. Exp Ther Med 13: 401-404, 2017.

10. Li MQ, Shi ZX, Xu JY, Lu B, Li JQ, Xu YJ, Wang XM, Li SM and Mo X: Hemodiafiltration combined with resin-mediated absorption as a therapy for hyperlipidemic acute pancreatitis. Cell Biochem Biophys 69: 699-702, 2014.

11. Wang GK, Zhu JQ, Zhang JT, Li Q, Li Y, He J, Qin YW and Jing Q: Circulating microRNA: A novel potential biomarker for early diagnosis of acute myocardial infarction in humans. Eur Heart J 31: 659-666, 2010.

12. Mukhadi S, Hull R, Mbita Z and Dlamini Z: The role of microRNAs in kidney disease. Noncoding RNA 1: 192-221, 2015.
13. Ji H, Tian D, Zhang B, Zhang Y, Yan D and Wu S: Overexpression of miR-155 in clear-cell renal cell carcinoma and its oncogenic effect through targeting FOXO3a. Exp Ther Med 13: 2286-2292, 2017.

14. Blaya D, Aguilar-Bravo B, Hao F, Casacuberta-Serra S, Coll M, Perea L, Vallverdú J, Graupera I, Pose E, Llovet L, et al: Expression of microRNA-155 in inflammatory cells modulates liver injury. Hepatology 68: 691-706, 2018.

15. Zhang W, Shi L, Zhang H, Wang C, Gao S, Ma Y, Li W, Liu J, Wang $J$ and Liu J: Effect of alprostadil on serum level of miRNA-155 in uremic patients. Zhong Nan Da Xue Xue Bao Yi Xue Ban 40: 735-741, 2015 (In Chinese).

16. Livak KJ and Schmittgen TD: Analysis of relative gene expression data using real-time quantitative PCR and the 2(-Delta Delta C(T)) method. Methods 25: 402-408, 2001.

17. Cai W, Chen WL and Fu HL: Effect of Muxiang shunqi pill on digestive disorders of prephase uremia patients and its mechanism. Zhongguo Zhong Xi Yi Jie He Za Zhi 37: 34-38, 2017 (In Chinese).

18. Xue W, Zhao Y, Yuan M and Zhao Z: Chinese herbal bath therapy for the treatment of uremic pruritus: Meta-analysis of randomized controlled trials. BMC Complement Altern Med 19: 103, 2019.

19. Lisowska-Myjak B: Uremic toxins and their effects on multiple organ systems. Nephron Clin Pract 128: 303-311, 2014.

20. Li G, Ma H, Yin Y and Wang J: CRP, IL-2 and TNF- $\alpha$ level in patients with uremia receiving hemodialysis. Mol Med Rep 17: 3350-3355, 2018.

21. Zhang J, Yuan Y, An X, Ouyang C, Ren H, Yang G, Yu X, Lv X, Zhang $\mathrm{B}$, Wang $\mathrm{N}$, et al: Comparison of combined blood purification techniques in treatment of dialysis patients with uraemic pruritus. Int J Clin Exp Med 9: 8563-8568, 2016.

22. Belarbia A, Nouira S, Sahtout W, Guedri Y and Achour A: Metabolic syndrome and chronic kidney disease. Saudi J Kidney Dis Transpl 26: 931-940, 2015.

23. Gao Y, Ma X, Yao Y, Li H, Fan Y, Zhang Y, Zhao C, Wang L, Ma M, Lei Z and Zhang X: miR-155 regulates the proliferation and invasion of clear cell renal cell carcinoma cells by targeting E2F2. Oncotarget 7: 20324-30337, 2016.

24. Kurowska-Stolarska M, Alivernini S, Ballantine LE, Asquith DL, Millar NL, Gilchrist DS, Reilly J, Ierna M, Fraser AR, Stolarski B, et al: MicroRNA-155 as a proinflammatory regulator in clinical and experimental arthritis. Proc Natl Acad Sci USA 108: 11193-11198, 2011.

25. Ulivi P, Canale M, Passardi A, Marisi G, Valgiusti M, Frassineti GL, Calistri D, Amadori D and Scarpi E: Circulating plasma levels of miR-20b, miR-29b and miR-155 as predictors of bevacizumab efficacy in patients with metastatic colorectal cancer. Int J Mol Sci 19: 307, 2018.

26. Liang S, Cai GY, Duan ZY, Liu SW, Wu J, Lv Y, Hou K, Li ZX, Zhang XG and Chen XM: Urinary sediment miRNAs reflect tubulointerstitial damage and therapeutic response in $\operatorname{IgA}$ nephropathy. BMC Nephrol 18: 63, 2017.

27. Marcucci G, Maharry KS, Metzeler KH, Volinia S, Wu YZ, Mrózek K, Nicolet D, Kohlschmidt J, Whitman SP, Mendler JH, et al: Clinical role of microRNAs in cytogenetically normal acute myeloid leukemia: miR-155 upregulation independently identifies high-risk patients. J Clin Oncol 31: 2086-2093, 2013.

28. Dung NH, Kien NT, Hai NTT, Cuong PT, Huong NTT, Quyen DBQ, Tuan NM, Ha DM, Kien TQ, Dung NTT, et al: Measuring serum beta2-microglobulin to predict long-term mortality in hemodialysis patients using low-flux dialyzer reuse. Ther Clin Risk Manag 15: 839-846, 2019.

29. Chen L, Fan D and Shi X: Comparison of the effects of different blood purification methods on removal of macromolecules in uremia. Chin J Primar Med Pharm 24: 1445-1449, 2017 (In Chinese).

30. Levine BS, Rodríguez M and Felsenfeld AJ: Serum calcium and bone: Effect of PTH, phosphate, vitamin D and uremia. Nefrologia 34: 658-669, 2014 (In English, Spanish).

31. Gao P, Ma JR, Zhao L, Pei GC, Shi GJ and Li L: Effect of high-flux and low-flux hemodialysis on the side metabolites and cytokines in patients with uremia. J Hainan Med Univ 23: 61-64, 2017.

32. Chen Y, Duan J, Guo J, Shang E, Tang Y, Qian Y, Tao W and Liu P: Yuanhuapine-induced intestinal and hepatotoxicity were correlated with disturbance of amino acids, lipids, carbohydrate metabolism and gut microflora function: A rat urine metabonomic study. J Chromatogr B Analyt Technol Biomed Life Sci 1026: 183-192, 2016. 
33. Carrero JJ and Stenvinkel P: Inflammation in end-stage renal disease-what have we learned in 10 years? Semin Dial 23: 498-509, 2010

34. Zhou H, Zhou D, Lu J, Wu C and Zhu Z: Effects of pre-cardiopulmonary bypass administration of dexmedetomidine on cardiac injuries and the inflammatory response in valve replacement surgery with a sevoflurane postconditioning protocol: A pilot study. J Cardiovasc Pharmacol 74: 91-97, 2019.

35. Shao QY, Wan C, Liu J and Zhang M: Effect of peritoneal dialysis on inflammatory factors, nutritional index and renal function in patients with chronic renal failure. J Hainan Med Univ 23: 87-90, 2017.
36. Xiangming LI, Zhang W and Shen D: The effect of hemodialysis filtration combined with blood perfusion in the treatment of patients with uremic encephalopathy. Chin J Primary Med Pharm 23: 457-459, 2016.

37. Wang LY, Yin DX, Zhang DL, Xu R, Cui WY and Liu WH Improvement and influencing factors of blood pressure control by nephrologist referral in chronic kidney disease patients in China: A cohort study. Int Urol Nephrol 45: 1345-1353, 2013.

38. Wang Y, Li J, Tong L, Zhang J, Zhai A, Xu K, Wei L and Chu M: The prognostic value of miR-21 and miR-155 in non-small-cell lung cancer: A meta-analysis. Jpn J Clin Oncol 43: 813-820, 2013. 